\title{
9-Aminofluorenium Cations: A New Group of anti-Aromatic Molecules
}

\author{
N. Tyutyulkov*, I. Kavrakova, P. Nikolov \\ Academy of Sciences, Institute of Organic Chemistry, 1040 Sofia, Bulgaria \\ F. Dietz
}

Sektion Chemie der Universität Leipzig, 7010 Leipzig, DDR

Z. Naturforsch. 44b, 1451-1452 (1989); received June 5, 1989

9-Aminofluorenium Cations, anti-Aromatic Molecules

Quantum-chemical and spectral studies on the protonated form of fluorenone-anil show that this compound has anti-aromatic character. Its real structure should be considered as: 9-amino derivative of the anti-aromatic fluorenium cation.

The present study is related to our theoretical investigations $[1-3]$ of diamino derivatives of the antiaromatic cyclopentadienyl cation (CPC). The theoretical results were confirmed with the brilliant synthesis of 6-amino-1,4-dimethyl-5,7-diphenyl1,2,3,4-tetrahydrocyclopentapyrazin-6-ylium-tetrafluoroborate, carried out by Gompper et al. [4] and of some other stable cyclopentadienyl salts [5]. The studies of Gompper [6] demonstrated also the possibility for the CPC derivatives to serve as building blocks-"bausteine" [6] for new materials.

The aim of this work, which has the character of a first preliminary report, is to show that the protonated forms of the anils of fluorenone have antiaromatic character.

The first theoretical considerations for carring out these investigations are the results from the ab initio studies of system 1 (applying STO-3 G basis set with geometry optimization). They show (see diagrams below) that from the alternative structures: classical $\mathbf{1 a}$ and anti-aromatic $\mathbf{1 b}$ the anti-aromatic structure corresponds to the real system:

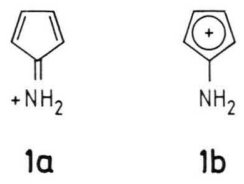

First of all this is confirmed by the characteristic for anti-aromatic systems [2, 3, 7, 8] geometry with marked bonds lengths alternation - a result of a second order Jan-Teller effect. It is seen from dia-

\footnotetext{
* Reprint requests to Prof. Dr. N. Tyutyulkov.
}

Verlag der Zeitschrift für Naturforschung, D-7400 Tübingen 0932-0776/89/1100-1451/\$ 01.00/0 grams below, where the bonds lengths in the cycle (in $\mathrm{pm}$ ) from our $a b$ initio studies of $\mathbf{1}$ are given, as well the results from the ab initio studies of Borden and Davidson [7] for the equilibrium - $A_{1}(1)$ Jan-Teller form of CPC:

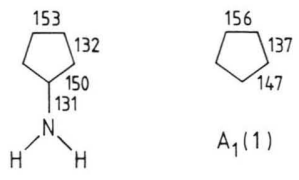

The CPC amino derivative $\mathbf{1}$ has not been reported, as it is most probably unstable at ordinary conditions. It should be expected that the anelation of the five-atom cycle will lead to relative stabilization of the corresponding derivative. Quite a long time ago (1910) Reddelien and Kliegl [9.10] take notice of the fact that the anils of fluorenone, being yellow as most anils, turn red in concentrated acids. That fact is of interest by itself, as protonation of anils is connected with hypsochromic effect [11]. The spectral characteristics of the red-coloured protonated forms of the anils of fluorenone have not been reported $[9,10]$, neither have they been investigated by other authors later.

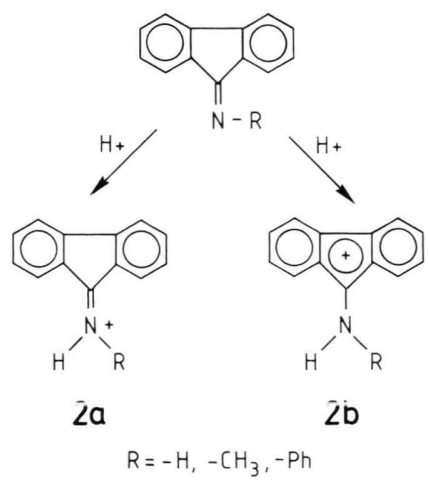


The results from the theoretical investigations of $\mathbf{1}$ give us grounds to suggest that from the two possible structures $\mathbf{2} \mathbf{a}$ and $\mathbf{2} \mathbf{b}$ the anti-aromatic structure $\mathbf{2 b}$ corresponds to the protonated forms of the imine and of the anil derivatives of fluorenone.

Optimization of the geometry of $\mathbf{2}$ when $\mathrm{R}=\mathrm{H}$ by the MINDO/3 method [12], while taking into account all conformational variables assuming a $\mathrm{C}_{2 \mathrm{v}}$ symmetry give the results, shown on the diagram:

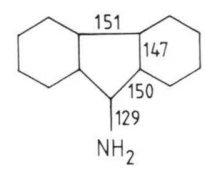

It can be seen, that as in $\mathbf{1}$, a considerable alternation of bonds lengths in the five-atom cycle exists.

The total $\pi$-electron charge of the five-atom cycle in $2(\mathrm{R}=\mathrm{H})$, calculated by the PPP-method is $\mathrm{Q}=$ 4.932 , the charge of the $\mathrm{N}^{\text {th }}$ atom $\mathrm{q}=1.490$.

The maxima of the longest-wavelength bands in the absorption spectrum of the protonated form of the anil derivative of fluorenone in $\mathrm{H}_{2} \mathrm{SO}_{4}(96 \%)$ are at: $\lambda_{1}=500 \mathrm{~nm}\left(\varepsilon=270 \mathrm{l} \cdot \mathrm{mol}^{-1} \cdot \mathrm{cm}^{-1}\right)$ and $\lambda_{2}=352 \mathrm{~nm}$ $\left(\varepsilon=12,000 \mathrm{l} \cdot \mathrm{mol} \cdot \mathrm{cm}^{-1}\right)$. In the electronic spectrum of the base (in $\mathrm{CHCl}_{3}$ ) there is no band at $500 \mathrm{~nm}$ (the latter being responsible for the red colour of the protonated form) and the longest-wavelength absorption bands are at $\lambda_{1}=388 \mathrm{~nm}\left(\varepsilon=2260 \mathrm{l} \cdot \mathrm{mol} \cdot \mathrm{cm}^{-1}\right)$ and $\lambda_{2}=299 \mathrm{~nm}\left(\varepsilon=11,290 \mathrm{l} \cdot \mathrm{mol} \cdot \mathrm{cm}^{-1}\right)$.

There is a good agreement between the absorption spectrum of the protonated form and the results of PPP-calculations: $\lambda_{1}=570 \mathrm{~nm}$ and $\lambda_{2}=399 \mathrm{~nm}$, the computed oscillator strengths are $\mathrm{f}_{1}=0.007$ and $\mathrm{f}_{2}=$ 0.495 , respectively.

This calculations are performed by the PPPmethod (SCF-CI); the configurational interaction includes all singly-excited configurations, using the parametrization in [13], the geometry is determined by the MINDO/3 method.

The conclusions for the anti-aromatic character of the protonated form of fluorenone-anil can be generalized also for the other anils of fluorenone, for which a bathochromic shift in concentrated acids has been reported $[9,10]$.

The authors express their gratitude to Mrs. M. Tasseva and Dr. V. Entchev for the help in the quantum-chemical calculations.
[1] A. Mehlhorn and J. Fabian, J. Mol. Struct. (Theochim.) 109, 27 (1984).

[2] N. Tyutyulkov, B. Kurtev, Yu. Stefanovsky, F. Dietz, J. Fabian, A. Mehlhorn, G. Olbrich, and O. E. Polansky, J. Mol. Struct. 114, 279 (1985).

[3] J. Fabian, A. Mehlhorn, and N. Tyutyulkov, J. Mol. Struct. 151, 355 (1987).

[4] R. Gompper and H. Glöckner, Angew. Chem. 96, 48 (1984).

[5] R. Gompper and T. Gessner, Angew. Chem. 97, 996 (1985).

[6] R. Gompper and H.-U. Wagner, Angew. Chem. 100, 1492 (1988).
[7] W. Th. Borden and E. Davidson, J. Am. Chem. Soc. 101, 3771 (1979).

[8] W. Th. Borden, Diradicals, J. Wiley, New York (1982).

[9] G. Raddelien, Berichte 43, 2476 (1910).

[10] A. Kliegl, Berichte 43, 2488 (1910).

[11] E. B. Agracheva, L. N. Kirichenko, and V. F. Gachkowski, Zh. Obstei Khimii (USSR) 44, 1303 (1974).

[12] R. C. Bingham, M. J. S. Dewar, and D. H. Lo, J. Am. Chem. Soc. 97, 1285 (1975).

[13] J. Griffiths, Dyes and Pigments 3, 2120 (1982). 